\title{
RCAN1 Regulates Vascular Branching during Xenopus laevis Angiogenesis
}

\author{
Masakazu Fujiwara $^{a}$ Takashi Hasebe $^{c}$ Mitsuko Kajita ${ }^{b}$ Atsuko Ishizuya-Oka ${ }^{c}$ \\ Mohammad Ghazizadeh $^{\mathrm{a}}$ Oichi Kawanami ${ }^{\mathrm{a}}$ \\ Departments of a Molecular Pathology and ${ }^{\mathrm{b}}$ Molecular Biology, Institute of Development and Aging Sciences, \\ Graduate School of Medicine, Nippon Medical School, and 'Department of Biology, Nippon Medical School, \\ Kawasaki, Japan
}

\section{Key Words}

Endothelial cells $\cdot$ Calcineurin $\cdot$ RCAN1 $\cdot$ DSCR1 $\cdot$ MCIP .

Vasculogenesis

\begin{abstract}
Background/Aims: The mechanisms that regulate the sizerelated morphologies of various blood vessels from the aorta to capillary vessels are still poorly understood. In this study, we evaluate the involvement of regulator of calcineurin 1 (RCAN1), a regulatory protein in the calcineurin/NFAT signal transduction pathway, in vascular morphology to gain further insight into these mechanisms. Methods and Results: We first generated 2 types of vasculature in vitro from the same source of human umbilical vein endothelial cells by fibrin gel assay. We found that RCAN1 was significantly upregulated in large vessels with low branching frequencies when compared with small vessels with high branching frequencies. Next, to clarify whether RCAN1 regulates the branching of blood vessels in vivo, we injected RCAN1 mRNA into fertilized Xenopus laevis eggs. Overexpression of RCAN1 decreased the number of branching points that sprouted from intersomitic vessels during $X$. laevis angiogenesis. In addition, coexpression of calcineurin $A$, a target of RCAN1,
\end{abstract}

could rescue RCAN1-suppressed vascular branching. Conclusions: These results provide in vivo evidence of RCAN1regulated vascular branching which may play a role in the patterning of morphologically different vasculature.

Copyright $\odot 2010$ S. Karger AG, Basel

\section{Introduction}

Pronounced morphological differences among blood vessels exist, as represented by the size difference between the aorta and capillary vessels. Diameter and branching frequency are easily distinguishable characteristics between these 2 vasculatures $[1,2]$. Although these characteristics are closely related to their function, their regulatory mechanisms are poorly understood. Uncovering these regulatory mechanisms will further the understanding of complex vascular patterning and may also lead to new angiogenic therapies that may induce blood vessels with the desired branching frequency and size.

Previous studies have shown that vascular endothelial growth factor (VEGF) is a key regulator of the branching frequency and size of vasculature [3, 4]. Fibrin gel assay,

\section{KARGER}

Fax +41613061234 E-Mail karger@karger.ch www.karger.com
Dr. Mohammad Ghazizadeh

Department of Molecular Pathology

Institute of Development and Aging Sciences, Nippon Medical School

1-396 Kosugi-cho, Nakahara-ku, Kawasaki, Kanagawa 211-8533 (Japan)

Tel. +81 44733 1823, Fax +81 44733 1293, E-Mail fuji@nms.ac.jp 
in which the morphological difference in the size of vasculatures is reproducible in vitro [5], has shown that Notch signaling regulates the branching frequency and diameter of vasculature by possibly interacting with growth factors such as VEGF [6]. However, little is known about VEGF-induced factors other than Notch signaling. Because vascular patterning is a complex phenomenon, numerous factors other than Notch signaling must be involved in the regulation of the branching frequency and size of the vasculature. To identify such factors, we performed microarray analysis and compared small vessels with high branching frequencies (SVHB) and large vessels with low branching frequencies (LVLB). These 2 vasculatures were formed from the same endothelial cells by fibrin gel assay. Among the upregulated genes, we found that an interesting candidate gene, regulator of calcineurin 1 (RCAN1), was predominantly expressed in LVLB (preliminary observation).

Human RCAN1, also known as Down syndrome critical region 1 , is located at chromosomal region 21q22.1q22.2; this is the main region of interest for genes involved in Down syndrome $[7,8]$. RCAN1 has an acidic domain, a serine-proline-rich motif, a putative DNAbinding domain, and a proline-rich region [9]. Subcellular localization of HA-tagged RCAN1 was observed in both the cytoplasm and the nucleus of human umbilical vein endothelial cells (HUVECs) and osteosarcoma U2OS cells $[10,11]$. In normal human tissues, RCAN1 is highly expressed in adult heart, brain, and skeletal muscle as well as in fetal brain and kidney [12]. Further complementing the observation that RCAN1 is expressed in highly vascularized tissues, RCAN1 is also expressed in HUVECs [13].

At the molecular level, RCAN1 inhibits the phosphatase activity of the heterodimeric protein calcineurin. Calcineurin is composed of a catalytic A subunit (CnA) and a regulatory $\mathrm{B}$ subunit $(\mathrm{CnB})$ [14], and it acts by dephosphorylating nuclear factor of activated $\mathrm{T}$ cells (NFAT) in a $\mathrm{Ca}^{+} /$calmodulin-dependent manner. Thus, an increase in calcineurin activity would lead to the nuclear entry of NFAT and the assembly of NFAT transcription complexes to induce gene expression [15]. RCAN1 inhibits cytoplasmic calcineurin/NFAT signaling by binding to $\mathrm{CnA}[16,17]$. A disruption of calcineurin/NFAT signaling causes developmental abnormalities in vasculatures and heart valves $[18,19]$. RCAN1 also functions as an endogenous inhibitor of VEGF/calcineurin-mediated angiogenesis in endothelial cells [20, 21]. These results again highlight the close relationship between RCAN1 and blood vessel formation. However, no study has demonstrated that RCAN1 truly regulates vascular morphology in vivo. One reason for this delay may be that transgenic mice overexpressing RCAN1 are lethal [22].

In the present study, we analyzed the influence of RCAN1 on vascular morphology. First, using 2 morphologically different vasculatures generated by fibrin gel assay, we confirmed a significant upregulation of RCAN1 expression in LVLB compared with SVHB. We also found a correlation between RCAN1 expression and vascular branching. Next, using the Xenopus laevis model, we demonstrated that the overexpression of RCAN1 suppressed vascular branching during embryogenesis in vivo. Moreover, this effect could be ameliorated by the coinjection of $\mathrm{CnA}$, a downstream target of RCAN1. These findings demonstrate a newly identified role of RCAN1 in vascular branching.

\section{Materials and Methods}

\section{Cell Culture and Fibrin Gel Assay}

HUVECs and normal human lung fibroblasts (NHLFs) were obtained from Clonetics (Walkersville, Md., USA). HUVECs were cultured in an EGM-2 BulletKit, and NHLFs were grown in an FGM-2 BulletKit routinely. Cells were plated onto gelatin-coated flasks and grown under $5 \% \mathrm{CO}_{2}$ at $37^{\circ} \mathrm{C}$. Only passages $3-7$ of each cell line were used for the experiments. Fibrin gel assay was conducted as described in detail by Nakatsu et al. $[5,23]$ with the minor modification of using NHLFs instead of skin fibroblasts. Images of HUVECs in the fibrin gel assay were captured with an Olympus IX71 microscope.

\section{Quantification of Vessels in vitro}

Vasculatures that exceeded a diameter that was twice the size of that of the bead were counted as the number of sprouts per Cytodex 3 bead. To measure the diameter of the vasculature, a circle with a diameter twice the size of that of the bead was digitally drawn, and the length between 2 points on the circle and within a segment of vasculature was measured using NIH ImageJ. The largest vasculature diameter at each Cytodex 3 bead was used for analysis. More than 20 Cytodex 3 beads were assessed for each condition.

\section{Real-Time RT-PCR}

Total RNA was extracted from HUVECs in fibrin gel using an RNeasy Mini Kit (QIAGEN, Hilden, Germany). Real-time RTPCR was performed on an ABI PRISM 7700 (Applied Biosystems, Foster City, Calif., USA). RNA (50 ng) was amplified using RNAdirect $^{\mathrm{TM}}$ SYBR Green real-time PCR Master Mix. The PCR conditions for RCAN1 and GAPDH were as follows: denaturing, $90^{\circ} \mathrm{C}$ for $30 \mathrm{~s}$; reverse transcription, $61^{\circ} \mathrm{C}$ for $20 \mathrm{~min}$; preheating, $95^{\circ} \mathrm{C}$ for $1 \mathrm{~min}$, and $45 \mathrm{cycles}$ of $95^{\circ} \mathrm{C}$ for $15 \mathrm{~s}$ and $60^{\circ} \mathrm{C}$ for $30 \mathrm{~s}$. The following primer pairs were used: RCAN1 forward, $5^{\prime}$-GAAGATGCGACCCCAGTCAT-3'; reverse, 5'-CGCTGCGTGCAATTCATACT-3'; and GAPDH forward, 5'-CCTGCACCACCAACTG- 
CTTA-3'; reverse, 5'-AGTGATGGCATGGACTGTGG-3'. Gene expression levels were quantified based on the threshold cycle calibrated to a standard curve and normalized to GAPDH expression. All data were calculated relative to LVLB at $8 \mathrm{~h}$. Melting curve data acquisition was from 60 to $95^{\circ} \mathrm{C}$ at $0.03^{\circ} \mathrm{C}$ per second. For each gene, real-time RT-PCR assay was performed 3 times with $\mathrm{n}=2$.

Animals

$X$. laevis adult frogs were purchased from Oh-uchi A.A.S. (Saitama, Japan). Fertilized eggs were prepared as previously described [24].

\section{Cloning of the X. laevis RCAN1 and CnA}

RCAN1 and CnA cDNA were obtained from Open Biosystems (Livermore, Calif., USA). cDNA was amplified using PrimeSTAR $^{\mathrm{TM}}$ HS (Takara Bio, Inc., Otsu, Japan), and PCR fragments were subcloned into pT7Ts [24] for mRNA synthesis. For the synthesis of green fluorescent protein (GFP), pT7Ts GFP was used [24].

\section{Phylogenetic Analysis}

The amino acid sequences of the various RCAN1 proteins were aligned using ClustalW2 (http://www.ebi.ac.uk/Tools/ clustalw2/index.html). The data were then analyzed using PHYLIP version 3.68 and the UPGMA method for phylogenetic analysis (http://evolution.genetics.washington.edu/phylip.html). A total of 1,000 bootstrap replicates were carried out to assess the robustness of the tree.

\section{RNA Microinjection}

Synthetic mRNA encoding RCAN1, CnA, or GFP was generated by mMESSAGE mMACHINE (Ambion, Austin, Tex., USA) and microinjected into 1-cell stage embryos. For coinjection experiments, $1 \mathrm{ng}$ of RCAN and 2 ng of CnA mRNA were used. The injected embryos were kept at $18^{\circ} \mathrm{C}$ in $0.1 \times$ Marc's modified ringers (MMR) supplemented with 6\% Ficoll 400 overnight and then in $0.1 \times$ MMR until they reached stage 24-25. For Morpholino knockdown experiments, Morpholino oligonucleotides (MOs) obtained from GeneTools, LLC were injected into 1-cell stage embryos. The sequences of the MOs were: antisense RCAN1 MO, 5'-AGCTTTCTCCATGCTGCGACTCTCC-3'; inverted control MO, 5' $5^{\prime}$-CCTCTCAGCGTCGTACCTCTTTCGA-3', and control MO containing 5 miss pairs 5'-AGGTTACTCCATCCTGCGAGTCTGC-3'. Antisense RCAN1 MO was targeted against 5' UTR. Prior to injection, the MOs were warmed to $65^{\circ} \mathrm{C}$ for $5 \mathrm{~min}$, which helped prevent clogging of the microinjection needles.

\section{Microangiography of X. laevis Larvae}

Larvae at stages 39/40-48 were anesthetized with $0.02 \%$ 3 -aminobenzoic acid ethyl ester in $0.1 \times \mathrm{MMR}$ and placed on agarose gel. A micromanipulator was used to inject a total of 140 $300 \mathrm{nl}$ of $25 \%$ red India ink into the heart ventricle using glass capillaries. Larvae were immediately analyzed with an Olympus SZX16 stereoscopic microscope with an RFP filter set. For the RCAN1 mRNA dose experiment, more than 100 vessels were measured for length and tortuosity using NIH Image as described by Stockmann et al. [25]. Using the same data, the number of branching points was also determined $(n>4)$. To quantify vessel branching, the number of multibranched points in 0.25 - $\mathrm{mm}^{2}$ areas was counted. At least 2 regions were quantified at each area: area A, regions anterior to the dorsal longitudinal anastomotic vessel (DLAV) fused into the posterior cardinal vein (PCV); area B, the region between the DLAV fused into the PCV to the 4 th ISV, and area C, the region between the 4 th and 8 th ISV. More than 100 regions were analyzed for each sample. $X$. laevis larvae were staged according to Nieuwkoop and Faber [26]. Animal rearing and treatment were performed according to the guidelines set by the Nippon Medical School Animal Use and Care Committee.

\section{Western Blot Analysis}

Lysates were prepared by homogenizing $X$. laevis embryos or larvae in SDS lysis buffer (50 mM Tris-HCl, pH 7.4, 1\% SDS, and $1 \mathrm{mM}$ DTT). After a brief sonication, an aliquot equivalent to 1 or 0.5 embryos/larvae was used for RCAN1 or CnA detection, respectively, and was separated on $12.5 \%$ Ready Gel (Bio-Rad Laboratories, Inc., Hercules, Calif., USA). Protein was transferred to a PVDF membrane, and blots were probed with anti-RCAN1 antibody (Abnova, Taipei, Taiwan), anti-pan-calcineurin A antibody (Cell Signaling Technology, Inc., Danvers, Mass., USA), or anti$\beta$-actin antibody (Abcam, Cambridge, UK). Antibodies were detected using HRP-conjugated goat anti-mouse IgG or HRP-conjugated anti-rabbit IgG. Membranes were treated with Pierce ECL Western Blotting Substrate (Pierce, Rockford, Ill., USA) and developed with a Hyperfilm ECM (GE Healthcare Bio-Sciences AB, Uppsala, Sweden).

\section{Immunohistochemistry}

Immunohistochemical staining was performed on sections from formalin-fixed $X$. laevis larvae using anti-RCAN1 antibody (Abnova) and a standard method described previously [27].

\section{Immunoprecipitation}

Immunoprecipitation (IP) buffer (50 mM Tris- $\mathrm{HCl}, \mathrm{pH} 7.4,1 \%$ Triton X-100, $100 \mathrm{~mm} \mathrm{NaCl}, 1 \mathrm{~mm} \beta$-glycerophosphate, $1 \mathrm{~mm}$ $\mathrm{NaF}, 1 \mathrm{mM} \mathrm{Na} \mathrm{VO}_{4}$, complete protease inhibitor cocktail) was added to the embryos. After mechanical homogenization, the lysate equivalent of 11 embryos was used for immunoprecipitation. These lysates were incubated with rabbit anti-pan-calcineurin $\mathrm{A}$ antibody at $4^{\circ} \mathrm{C}$ overnight. Calcineurin was precipitated with nProtein A Sepharose 4 Fast Flow (GE Healthcare Bio-Sciences $\mathrm{AB})$. The beads were washed 5 times with $0.5 \mathrm{ml}$ IP buffer. All steps were carried out using ice-cold buffers. After being washed, the beads were resuspended in $50 \mu$ l SDS-PAGE sample buffer, and $15 \mu \mathrm{l}$ of each sample was separated on $12.5 \%$ Ready Gel. Detection of RCAN1 and CnA was conducted as described in the western blot analysis.

\section{Treatment with the Calcineurin Inhibitors}

Calcineurin inhibitors FK506 and cyclosporine A (CsA), were obtained from Sigma (St. Louis, Mo., USA) and were dissolved in $83 \%$ ethanol and $17 \%$ Tween 20 . Stage 46 larvae were treated with FK506 or CsA (5-100 nM) for 6 h every other day for a total of 3 times. After each treatment, the larvae were washed 3 times in $0.1 \times$ MMR for $10 \mathrm{~min}$ each. After the final wash, microangiography was performed. All final test solutions of the drugs contained $0.0415 \%$ ethanol and $0.0085 \%$ Tween 20 , including the controls. 

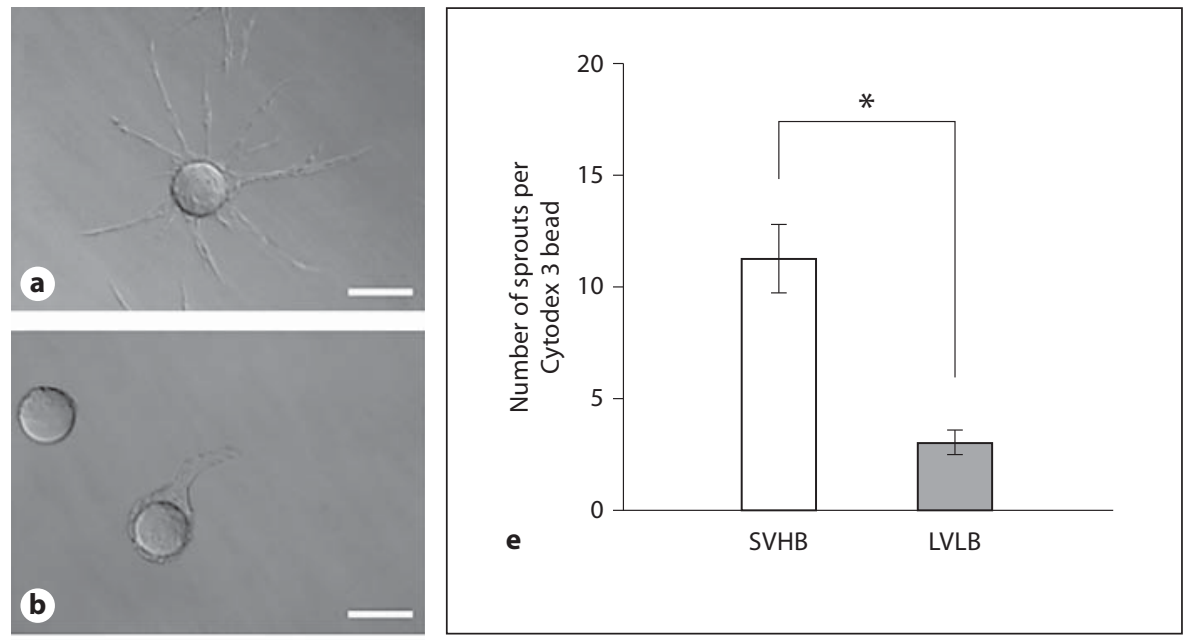

Fig. 1. The number of sprouts and diameters differ in the 2 morphologically different vasculatures generated from HUVECs. a, c Representative phase contrast micrographs of SVHB and (b, d) LVLB. SVHB and LVLB were treated with 2.5 or $15 \mathrm{ng} /$ $\mathrm{ml}$ VEGF, respectively, for 6 days. e The number of sprouts protruding from Cytodex 3 beads was counted and compared $\left({ }^{*} \mathrm{p}<0.05\right)$. f The sprouts with the largest diameter were compared between the small and large vasculatures using ImageJ software. ${ }^{*} \mathrm{p}<0.05$. Bars $=200 \mu \mathrm{m}$.
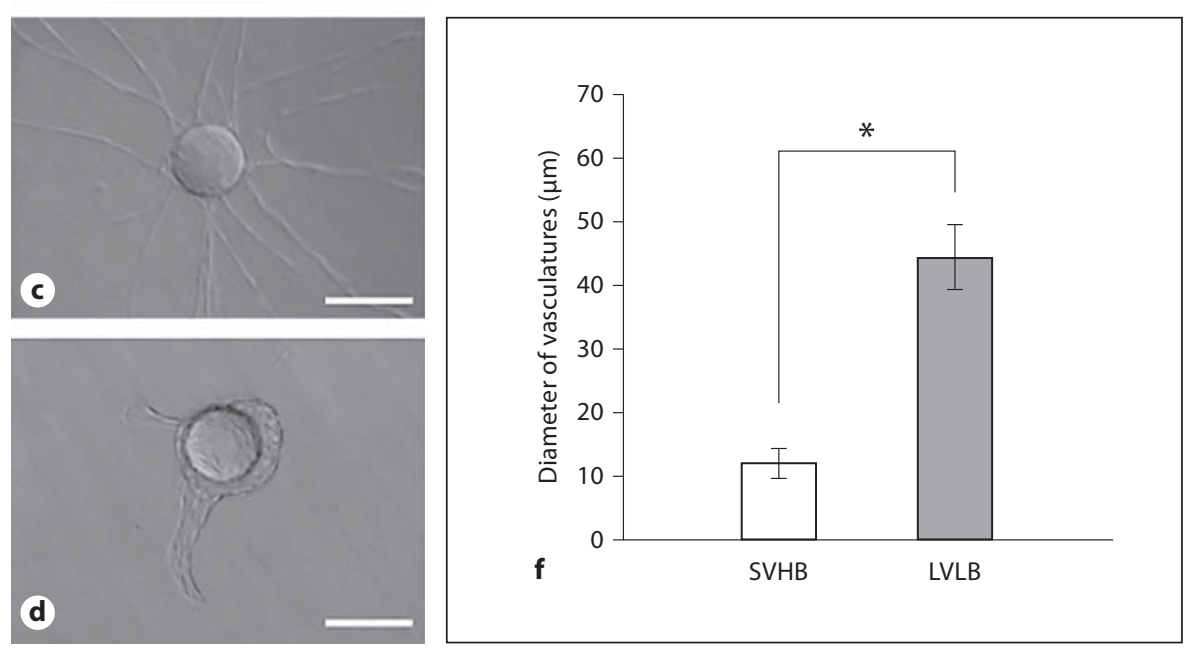

Statistical Analysis

All results are presented as means \pm SD. Differences between 2 groups were analyzed using Student's t test (2-tailed, equal variance). Comparisons between the mean values of multiple groups were analyzed using a 1-way ANOVA followed by Bonferroni's $t$ test for multiple comparisons. $\mathrm{p}<0.05$ was considered statistically significant. SPSS 14.0 (SPSS, Inc., Chicago, Ill., USA) was used to perform all statistical analyses.

\section{Results}

Generation of 2 Types of Vasculature with Different Branching Frequencies and Diameters

HUVECs cultured on Cytodex 3 beads were embedded in fibrin gel for 6 days. In the presence of 2.5 or 15 $\mathrm{ng} / \mathrm{ml}$ VEGF, the HUVECs formed distinctively different shapes (fig. 1a, b). HUVECs treated with $2.5 \mathrm{ng} / \mathrm{ml}$ VEGF showed more sprout formation (fig. 1c), whereas HUVECs treated with $15 \mathrm{ng} / \mathrm{ml}$ VEGF had fewer sprouts (fig. 1d). Furthermore, the vasculature treated with 2.5 $\mathrm{ng} / \mathrm{ml}$ VEGF had a smaller diameter (fig. 1c) than the vasculature that was treated with $15 \mathrm{ng} / \mathrm{ml}$ VEGF (fig. 1d). The number of sprouts was $11.3 \pm 3.1$ in the small vasculature and $3.1 \pm 1.2$ in the large vasculature (fig. 1e). The average diameter was $12.1 \pm 4.7 \mu \mathrm{m}$ in the small vasculature and $44.4 \pm 10.3 \mu \mathrm{m}$ in the large vasculature (fig. 1f). Hence, HUVECs treated with $2.5 \mathrm{ng} / \mathrm{ml}$ VEGF represented an SVHB and HUVECs treated with $15 \mathrm{ng} /$ ml VEGF represented an LVLB.

\section{Expression of RCAN1 Differs between SVHB and LVLB Fibrin Gel Assays}

Our microarray analyses of SVHB and LVLB generated from the same HUVECs showed that RCAN1 was 
highly expressed in LVLB but not in SVHB (preliminary observation). To confirm this observation, we examined RCAN1 mRNA expression using real-time quantitative RT-PCR (fig. 2a). The difference in RCAN1 expression between SVHB and LVLB became apparent $8 \mathrm{~h}$ after the start of fibrin gel assay. Specifically, the level of RCAN1 expression normalized by GAPDH was $0.25 \pm 0.07$ in SVHB relative to $1.00 \pm 0.13$ in LVLB after $8 \mathrm{~h}$ (fig. $2 \mathrm{a}$ ). After $24 \mathrm{~h}$ of culture, the RCAN1 level increased to 0.57 \pm 0.01 in SVHB and to $5.44 \pm 1.00$ in LVLB (fig. 2a).

\section{Alteration of RCAN1 Expression Is Followed by} Morphological Vasculature Changes in Sprouts

To further examine the association between vasculature morphology and the expression level of RCAN1, we altered the concentration of VEGF in a full-grown vasculature that had been cultured in fibrin gel for 6 days (fig. 2c). Figure $2 \mathrm{~b}$ shows that, at this time point (set as day 0 ), the 2 vasculatures still had distinct morphological features and distinct RCAN1 expression levels [0.56 \pm 0.19 in SVHB and $2.71 \pm 1.08$ in LVLB (fig. 2a)].

Eight hours after the concentration of VEGF had been switched from 2.5 to $15 \mathrm{ng} / \mathrm{ml}$, the expression level of RCAN1 increased from $0.56 \pm 0.19$ to $1.11 \pm 0.13$ (fig. 2 b; SVHB to LVLB). In contrast, switching the VEGF concentration from 15 to $2.5 \mathrm{ng} / \mathrm{ml}$ (LVLB to SVHB) decreased the expression level of RCAN1 (from $2.71 \pm 1.08$ to $1.52 \pm 0.10$ ). The difference in RCAN1 expression between the 2 vasculatures was further diminished at $24 \mathrm{~h}$ and reached the same level $48 \mathrm{~h}$ after the VEGF concentration was altered (fig. 2b). During these changes in RCAN1 expression levels, sprouts fused at the trunk region of SVHB treated with $15 \mathrm{ng} / \mathrm{ml}$ of VEGF (fig. 2c, SVHB to LVLB, arrows). On the other hand, splits were formed at the tip of the LVLB treated with $2.5 \mathrm{ng} / \mathrm{ml}$ of VEGF (fig. 2c, LVLB to SVHB, arrows).

Seventy-two hours after the VEGF treatment had been switched, the RCAN1 expression level was reversed in the 2 vasculatures (fig. 2b). As detected by real-time RT-PCR, the expression level of RCAN1 was $1.02 \pm 0.19$ in the vasculature treated with $15 \mathrm{ng} / \mathrm{ml}$ (SVHB to LVLB) and 0.66 \pm 0.14 in the vasculature treated with $2.5 \mathrm{ng} / \mathrm{ml}$ (LVLB to SVHB). The tips of vasculatures with a lower RCAN1 expression (LVLB to SVHB) were well branched, whereas vasculatures with a higher RCAN1 expression (SVHB to LVLB) did not have branched tips at day 6 (fig. 2c). These results indicated that the RCAN1 expression level was most closely correlated with the branching frequency of vasculatures and prompted us to investigate the RCAN1 function in vivo.

\section{Developmental Expression of RCAN1 mRNA} and Its Relationship with Vascular Branching during $\mathrm{X}$. laevis Embryogenesis

To determine the effect of RCAN1 expression on vascular morphology, we used $X$. laevis as an in vivo model. RCAN1 mRNA expression was weakly detected by RTPCR in unfertilized eggs, in embryos at stage 41, and later during X. laevis embryogenesis (fig. 3a). A trace amount of RCAN1 expression was detected between stages 33 and 40 (fig. 3b). Because RCAN1 was undetectable by western blotting in uninjected embryos/larvae (fig. 4), 38 cycles of amplification were required for the detection. In contrast, $\mathrm{CnA}$, a protein phosphatase that binds with RCAN1 [11], was detected at all stages examined using 28 cycles of PCR. The internal control, histone H4, was uniformly expressed at each stage, and there were no PCR products in H4 RT (-) samples (fig. 3a, b).

Branching of intersomitic vessels was not observed by microangiography until stages $39 / 40$ (fig. 3c, d); following the start of RCAN1 expression at stage 41 , they became detectable at stage 44 (fig. 3e, f). Although the branching and complexity of these vascular morphologies were still immature, many short sprouts of capillary vessels existed at this stage (fig. $3 \mathrm{~g}, \mathrm{~h}$ ). Thus, the bifurcation of microvessels sprouting from intersegmental vessels temporally coincides with RCAN1 expression during the normal development of $X$. laevis larvae.

Furthermore, to demonstrate that RCAN1 is indeed expressed in subpopulations of the embryonic vasculature that display low branching frequencies, immunohistochemical staining was performed. RCAN1 expression was mainly observed in large vessels representing vasculature with low branching frequencies (fig. 3i).

Fig. 2. The expression level of RCAN1 differs between the 2 morphologically distinct vasculatures. a Real-time RT-PCR analysis of RCAN1 mRNA expression in vascular models for SVHB and LVLB. RCAN1 expression relative to LVLB at $8 \mathrm{~h}$ was compared 8 and $24 \mathrm{~h}$ after starting the fibrin gel assay $\left({ }^{*} \mathrm{p}<0.05\right)$. b Time course of RCAN1 expression after switching the VEGF concentration of SVHB to LVLB (from 2.5 to $15 \mathrm{ng} / \mathrm{ml}$ ) and of LVLB to SVHB (from 15 to $2.5 \mathrm{ng} / \mathrm{ml}$ ). Expression of RCAN1 and GAPDH mRNAs was detected after $0,8,24,48$, and $72 \mathrm{~h}$ of treatment $\left({ }^{*} \mathrm{p}<0.05\right)$. c Morphology of vascular models after switching the concentrations of VEGF. Photomicrographs were taken 0,2 , and 6 days after switching the treatment condition. Arrows indicate morphological changes in the branching of sprouts. Bars $=200$ $\mu \mathrm{m}$. 


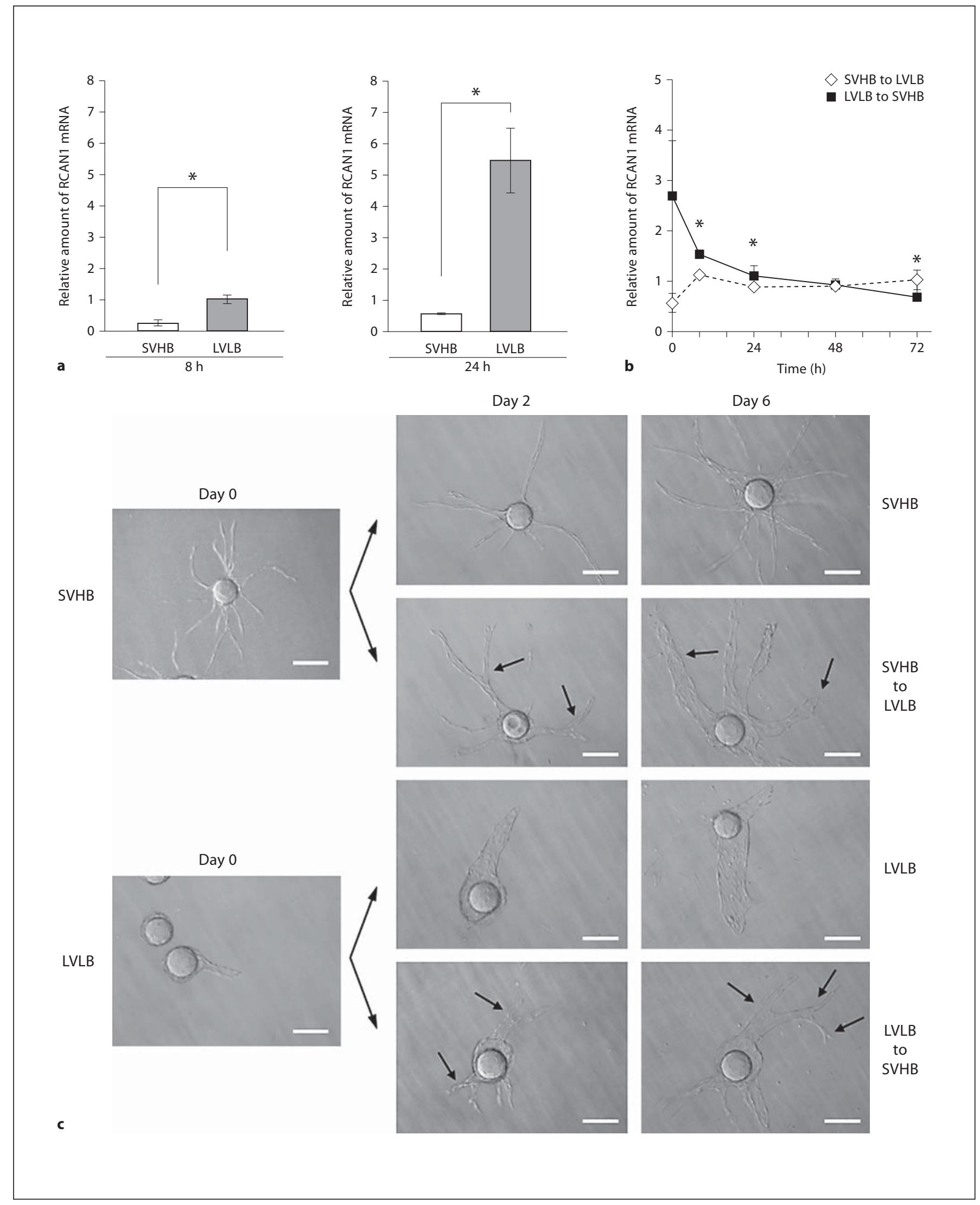




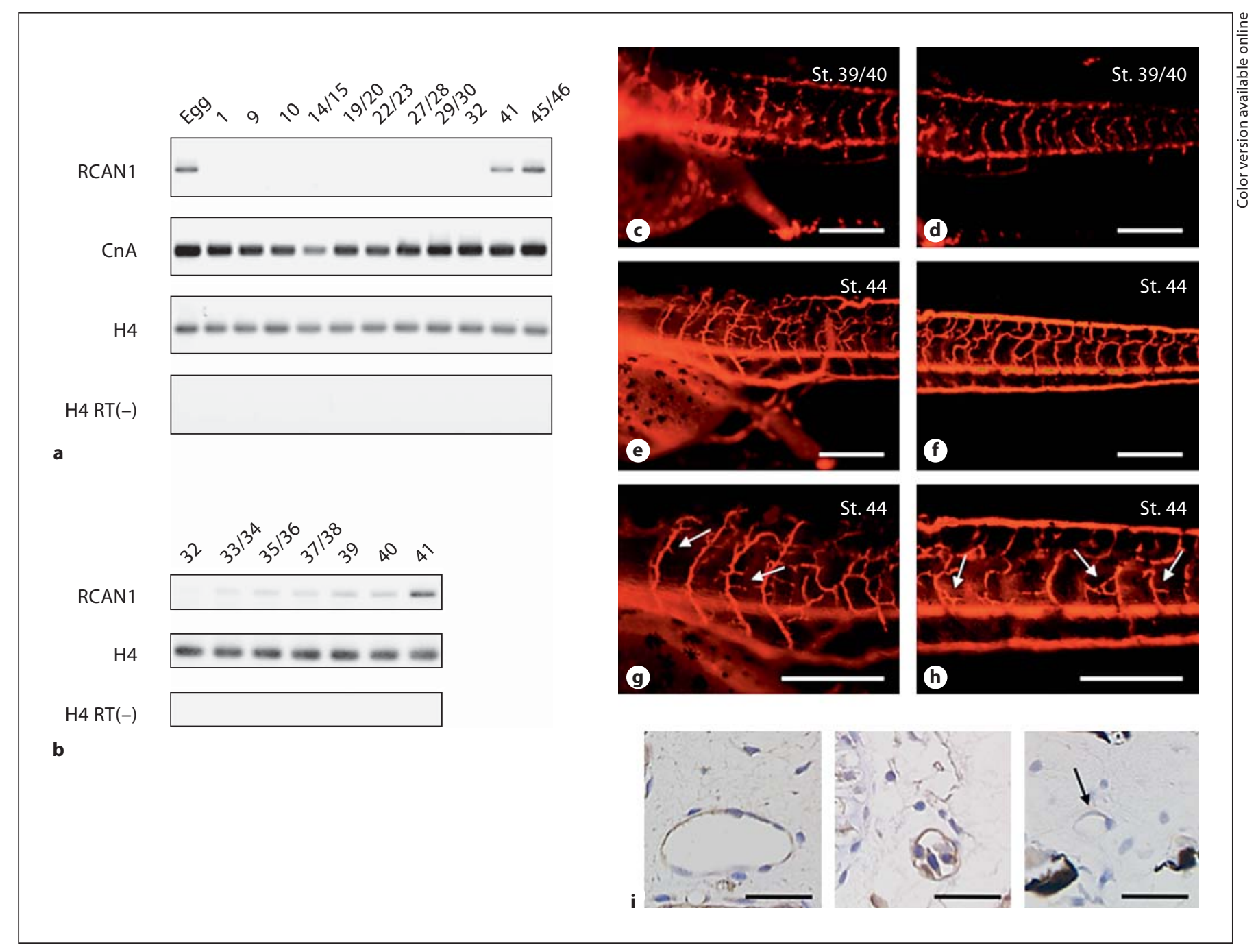

Fig. 3. Expression of RCAN1 and microvessel branching in a wildtype $X$. laevis larvae trunk. a Total RNA from different developmental stages was used for RT-PCR analysis. X. laevis embryonic stages are indicated at the top of each lane [26]. Amplified RCAN1 and CnA RT-PCR products were detected using $2 \%$ agarose gel. Histone $\mathrm{H} 4$ was used for normalization. H4 RT(-) was used as a negative control for DNA contamination. b RT-PCR showing ex- pressions of RCAN1 and H4 in stage 32-41 X. laevis. Microangiography of the wild-type larvae at stage $39 / 40$ (c, d) and stage 44 $(\mathbf{e}, \mathbf{f}) . \mathbf{g}, \mathbf{h}$ Magnified images of the larvae at stage 44 . Arrows indicate capillaries that are sprouting from intersomitic vessels. Bars $=500 \mu \mathrm{m}$. i Immunohistochemical expression of RCAN1 in the large vessels. Note the negative staining in the small vessels (arrow). St. $=$ Stage. Bars $=30 \mu \mathrm{m}$.
Suppression of Vascular Branching in RCAN1 $m R N A-I n j e c t e d \mathrm{X}$. laevis Larvae

RCAN1 cloned from $X$. laevis encodes 231 amino acids and shares $74 \%$ of its identity with mouse RCAN1 variant 2 (mRCAN1-4), 73\% with human RCAN1 variant 3 (hRCAN1-4), 71\% with mouse RCAN1 variant 1 (mRCAN1-1), and 70\% with human RCAN1 variant 1 (hRCAN1-1) (fig. 5). Phylogenetic analysis indicates that $X$. laevis RCAN1 belongs neither to the RCAN1-1 nor to the RCAN1-4 splicing variants in humans and mice but is similar to both variants (fig. 5b).

When $0.25-2.0$ ng of synthesized RCAN1 mRNA was injected into $X$. laevis-fertilized eggs, the animals showed a temporal development lag at late neurula stages but survived after the embryonic period. At stage 47 in which prominent vascular development is appreciated, the length of the microvessels was affected by RCAN1 and was longer in a dose-dependent manner as compared to 
Fig. 4. mRNA-injected X. laevis embryos/ larvae express high levels of RCAN1 and/ or CnA. a Western blot analysis of stage 9/10 embryos and stage 47/48 larvae injected with various concentrations of RCAN1 mRNA at the single-cell stage. $\mathbf{b}$ Extracts of uninjected and GFP-, RCAN1-, RCAN1/ CnA-, and CnA mRNA-injected embryos and larvae from the indicated stages were detected with an anti-human RCAN1 or CnA antibody.

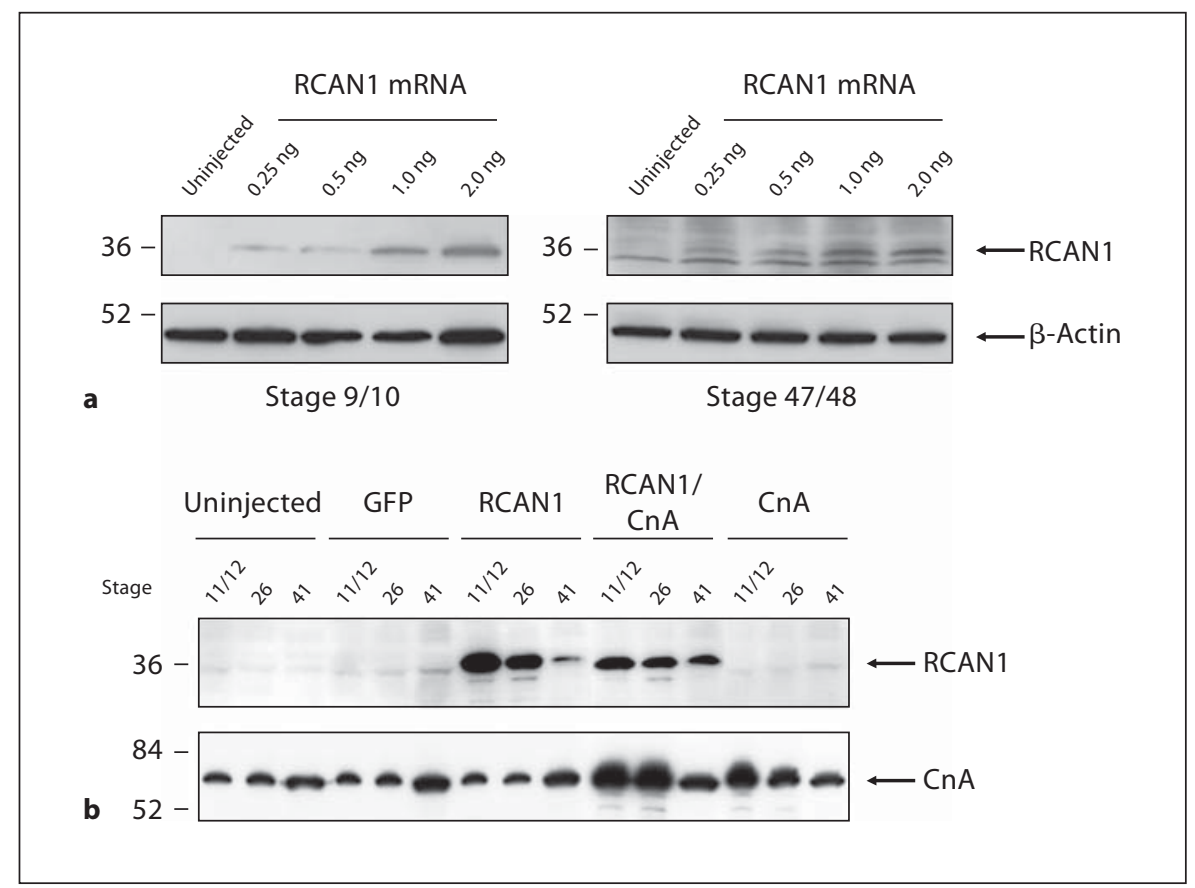

the uninjected controls (fig. 6a). Although the tortuousness of the vessels was not affected (fig. 6b), the number of branching points was significantly decreased in larvae that received 1.0 and $2.0 \mathrm{ng}$ of RCAN1 mRNA (fig. 6c). Therefore, the number of branching points was further analyzed with a GFP mRNA-injected control. As depicted by microangiography, injection of $1.0 \mathrm{ng}$ of RCAN1 mRNA decreased the branching frequency, and microvessels sprouting from intersegmental vessels were poorly branched (fig. $6 \mathrm{~h}, \mathrm{i})$. In uninjected (fig. $6 \mathrm{~d}$, e) and GFP mRNA-injected larvae (fig. 6f, g), zigzag networks of blood vessels and small areas surrounded by microvessels were observed; these patterns (fig. 6, inset: asterisk) were rarely observed in RCAN1 mRNA-injected larvae.

To further quantify the branching frequency, the number of branching points was counted in 3 different areas (fig. 6n). This number was significantly reduced compared to uninjected and GFP-injected larvae in area A (region anterior to the DLAV fused into PCV), area B (region between DLAV fused into PCV to the 4th ISV), and area $\mathrm{C}$ (region between the 4th and 8th ISV) of RCAN1-injected larvae (fig. 6j-1). An attenuation of bifurcation was observed in a wide area of the trunk in response to RCAN1 mRNA. The average number of branching points in all 3 areas was $21.0 \pm 4.1$ in uninjected larvae, $19.4 \pm 4.1$ in GFP-injected larvae, and $13.0 \pm 3.6$ in RCAN1-injected larvae (fig. 6m).

RCAN1 Regulates Vascular Branching
To confirm whether RCAN1 mRNA was properly translated into protein and persistently expressed, western blotting was performed (fig. 4). RCAN1 was expressed in proportion to the amount of mRNA injected at the proper product size (stages $9 / 10$ and 47/48) (fig. 4a), and was detected at all stages examined (stages 11/12, 26, and 41) (fig. 4b). In contrast, endogenous RCAN1 was not detected in uninjected or GFP-injected embryos/larvae (fig. 4b).

\section{Coexpressed CnA Interacts with RCAN1 and}

Rescues RCAN1-Suppressed Vascular Branching

Because RCAN1, also known as calcineurin-interacting protein (MCIP), interacts with $\mathrm{CnA}$ and inhibits its phosphatase activity, we investigated whether the coexpression of CnA could inhibit the effect of RCAN1 upon vascular branching (fig. 7). The number of microvessels sprouting from intersegmental vessles was reduced in RCAN1-injected larvae as mentioned above. However, when $2.0 \mathrm{ng}$ of CnA mRNA was coinjected into $X$. laevis eggs with 1.0 ng of RCAN1 mRNA, the reduced branching frequency recovered to the levels of the uninjected and GFP-injected control larvae (fig. 7a, b). Branching of microvessels in the CnA-injected larvae was similar to that in the controls. The average numbers of branching points were $18.5 \pm 4.8,18.7 \pm 4.7,13.4 \pm 4.3,17.4 \pm 5.6$, and $16.1 \pm 5.1$ in uninjected and GFP-, RCAN1-, CnA-, 
Fig. 5. Alignment and phylogeny of RCAN1. a Alignment of the amino acid sequences of RCAN1. Residues shaded in black are identical in $>50 \%$ of the predicted proteins; similar residues are highlighted in gray. $X$. laevis RCAN1 (xRCAN1, GenBank accession No. BC044332), mouse RCAN1 variant 2 (mRCAN1-4, GenBank accession No. NM_019466), rat RCAN1 (rRCAN1, GenBank accession No. NM_153724), human RCAN1 variant 3 (hRCAN1-4, GenBank accession No. NM_203418), mouse RCAN1 variant 1 (mRCAN1-1, GenBank accession No. NM_001081549), human RCAN1 variant 1 (hRCAN1-1, GenBank accession No. NM_004414), zebrafish RCAN1 (zRCAN̄1, GenBank accession No. XM_001922343), Caenorhabditis elegans RCAN1 (cRCN-1, GenBank accession No. NM_065822), and fly RCAN1 (dRCAN1/ Sarah, GenBank accession No. NM_079654) proteins are depicted. b Phylogenetic analysis of the $X$. laevis amino acid sequences with sequences from other species. Distance analysis with the UPGMA method was performed. The bootstrap values of each branch are indicated. The percentage of amino acids identitical to $X$. laevis RCAN1 (xRCAN1) is also indicated.
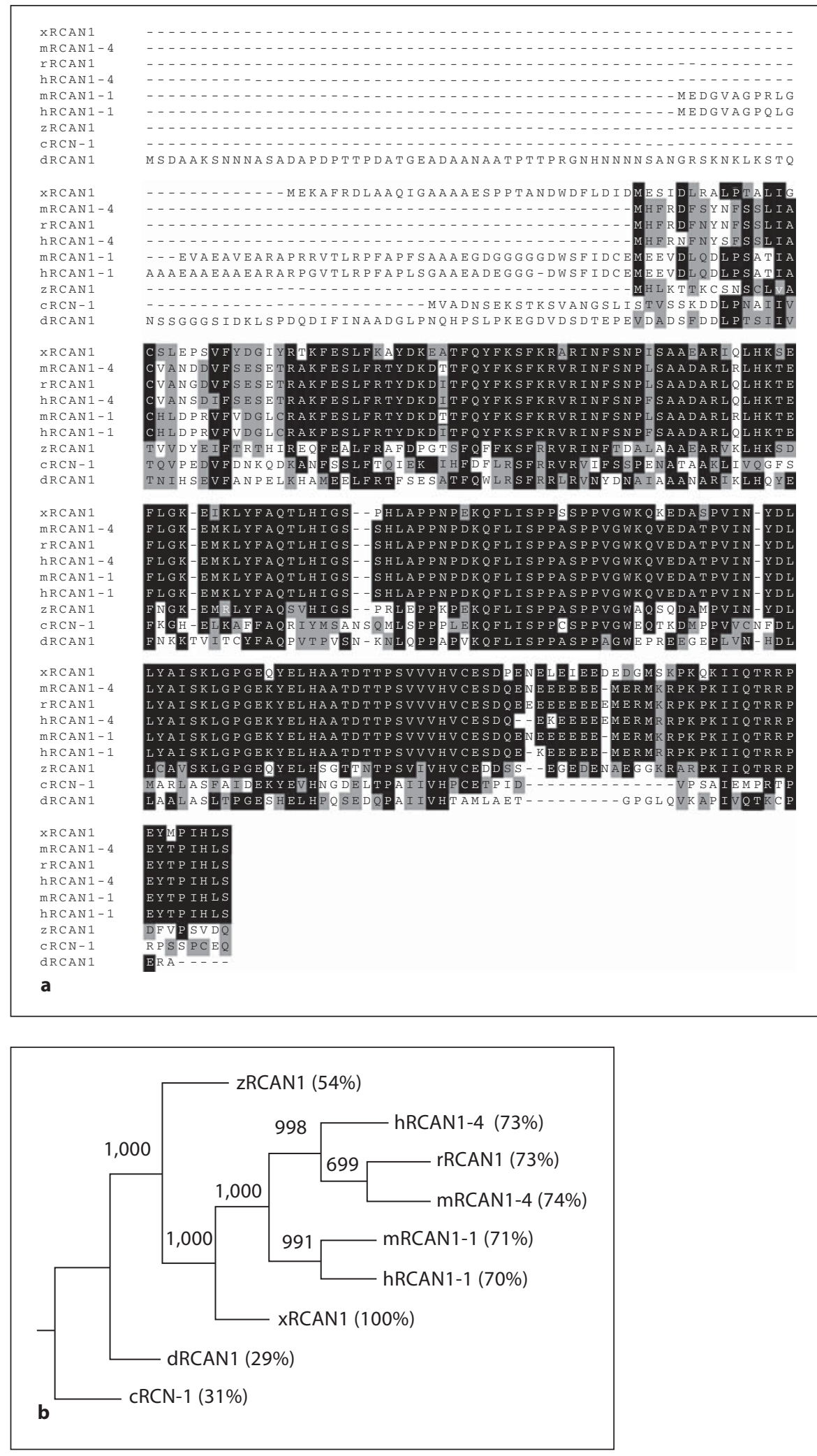


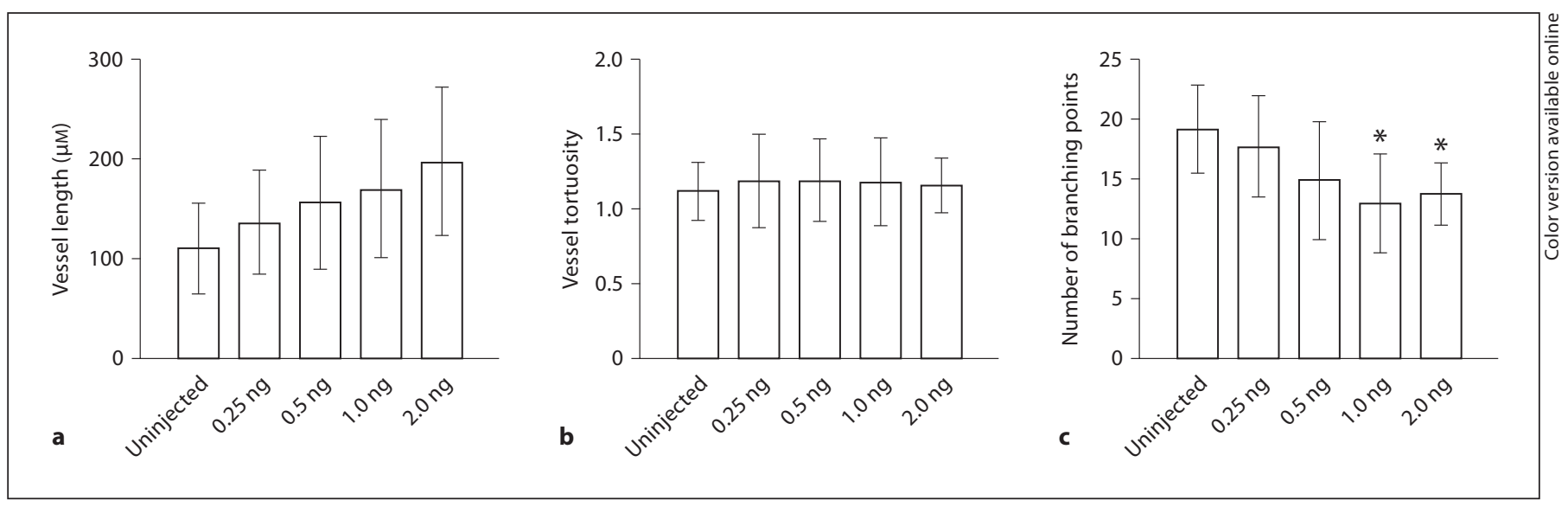

Fig. 6. Overexpression of RCAN1 in X. laevis larvae suppresses the branching of capillaries sprouting from intersegmental vessels. Effect of various doses of RCAN1 mRNA on vessel length (a), vessel tortuosity (b), and number of branching points (c) $\left(^{*} \mathrm{p}<\right.$ 0.05). Microangiography in stage $47 / 48$ of the uninjected control $(\mathbf{d}, \mathbf{e})$ and of the GFP mRNA-injected $(\mathbf{f}, \mathbf{g})$ and RCAN1 mRNAinjected $(\mathbf{h}, \mathbf{i})$ larvae. The insets show an enlarged view of vascular branching in an area of $0.15 \mathrm{~mm}^{2}$. The asterisk denotes a small area enclosed by microvessels. Bars $=500 \mu \mathrm{m}$.
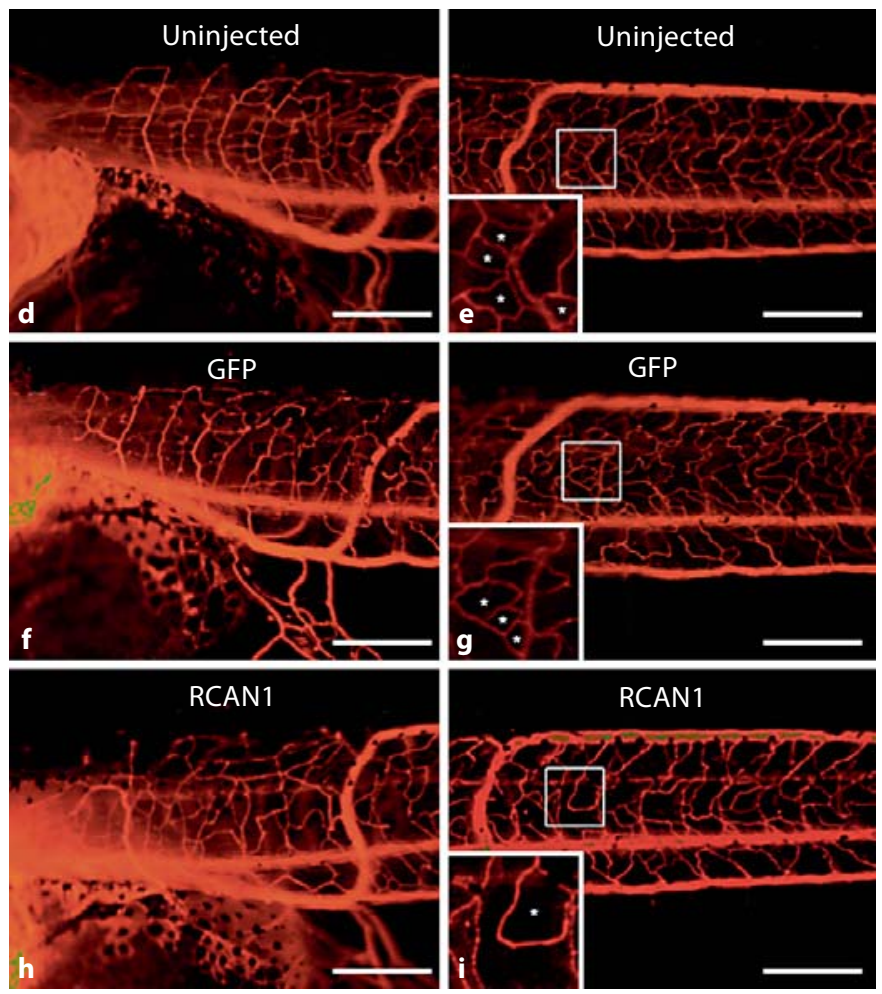

and RCAN1/CnA-injected larvae, respectively (fig. 7b). Compared with the RCAN1-injected larvae, the other experimental groups showed a significantly higher number of branching points $(\mathrm{p}<0.05)$. In contrast, no significant difference was observed in the body size of $X$. laevis larvae among the groups. The full-lengths of the larvae were $13.0 \pm 1.0,13.5 \pm 0.9,12.6 \pm 0.9,13.1 \pm 0.9$, and 13.1 $\pm 0.8 \mathrm{~mm}$ in uninjected and GFP-, RCAN1-, CnA-, and RCAN1/CnA-injected larvae, respectively. Western blot analysis confirmed the expression of these exogenous

proteins in embryos or larvae injected with the indicated mRNAs (fig. 4b).

Next, to confirm that CnA interacted with RCAN1 and inhibited the effect of RCAN1 in vivo, immunoprecipitation was carried out (fig. 7c). The immunoprecipitation of $\mathrm{CnA}$ from the extracts of RCAN1/CnA-injected embryos as well as of RCAN1-injected embryos led to the coprecipitation of RCAN1. RCAN1 was not detected in uninjected or GFP- or CnA mRNA-injected embryo extracts. We also confirmed that $\mathrm{CnA}$ was precipitated with 


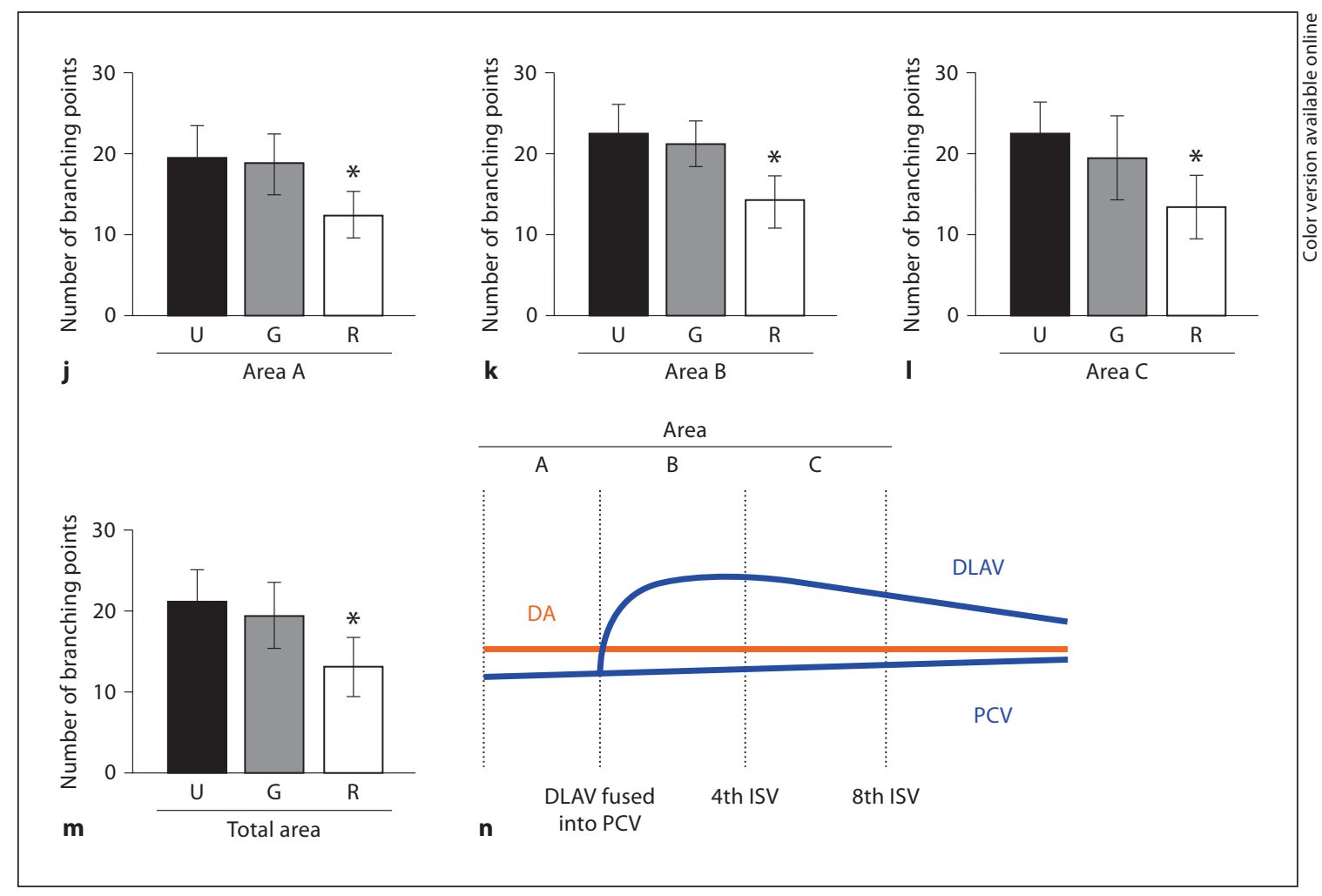

Fig. 6. Overexpression of RCAN1 in X. laevis larvae suppresses the branching of capillaries sprouting from intersegmental vessels. The number of vascular branching points at areas A (j), B (k), and C (I) as well as the total area $(\mathbf{m})\left({ }^{*} \mathrm{p}<0.05\right)$. $\mathrm{U}=$ Uninjected; $\mathrm{G}=\mathrm{GFP} ; \mathrm{R}=\mathrm{RCAN1} \mathbf{n}$ Diagram depicting areas A-C which were selected for the analysis of the vascular branching points.

nProtein A Sepharose 4 Fast Flow conjugated with rabbit anti-pan-calcineurin A antibody. These results indicate that CnA binds to RCAN1 in vivo and support our model that RCAN1 suppresses vascular branching by interacting with calcineurin.

\section{Calcineurin Inhibitors FK506 and CsA Suppress}

Vascular Branching in X. laevis Larvae

A pharmacological approach was taken to demonstrate that the inhibition of calcineurin has a negative effect on vascular branching as shown by RCAN1 overexpression. When $X$. laevis larvae were exposed to calcineurin inhibitors at stage 46, both FK506 and CsA inhibited vascular branching as shown in area A (fig. 7d). An effect of FK506 on vascular branching was observed at $50 \mathrm{nM}$ or more. On the other hand, an inhibitory effect of CsA was observed at $100 \mathrm{nM}$. At $5 \mathrm{nM}$, neither FK506 nor CsA inhibited vascular branching, and the vascular branching was at the same level as that of the untreated or control solvent.

\section{Discussion}

Previous microarray analyses reported RCAN1 to be one of the most strongly induced genes in response to VEGF treatment in several types of human endothelial cells $[10,13,21]$. Although the involvement of RCAN1 in angiogenesis has been frequently shown [20,28], little attention has been directed to date to the role of RCAN1 in vascular branching. In the present study, we performed both in vitro and in vivo experiments to examine the effect of RCAN1 on vascular formation, focusing especially on branching. In vitro vascular models for SVHB and LVLB showed that the expression of RCAN1 is correlated with the frequency of vascular branching. More importantly, we demonstrated that the overexpression of RCAN1 suppressed microvessel branching in X. laevis larvae. Our results provide the first in vivo evidence that RCAN1 suppresses vascular branching.

Because of the lethality of transgenic mice overexpressing RCAN1 [22], it has been difficult to study the 


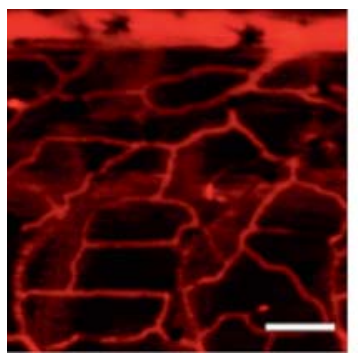

a

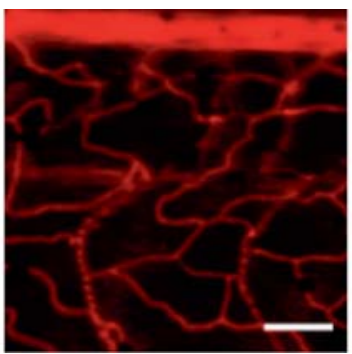

GFP

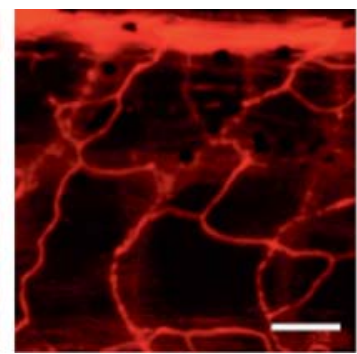

RCAN1

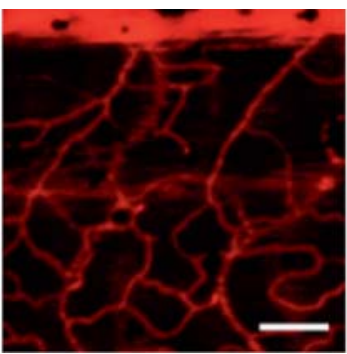

$\operatorname{CnA}$

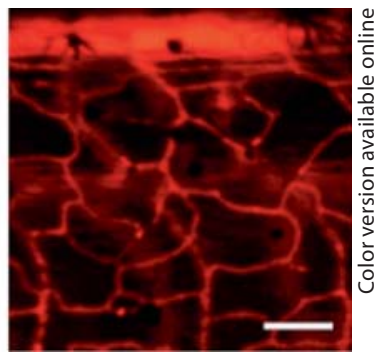

RCAN1/CnA

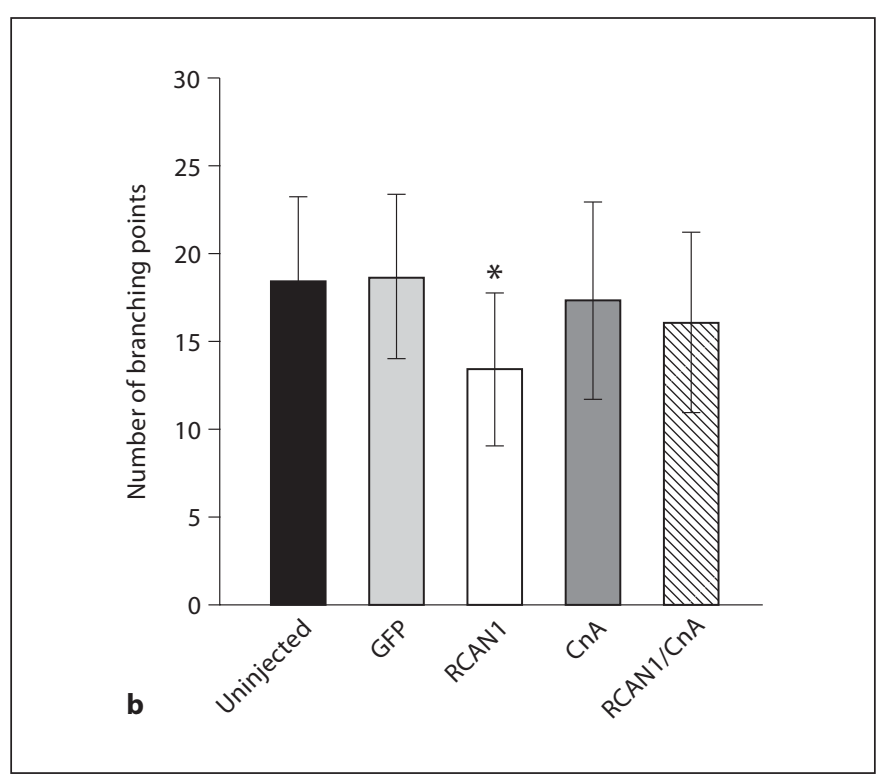

Fig. 7. RCAN1 suppresses vascular branching by interacting with calcineurin. a Microangiography of uninjected and GFP-, RCAN1-, CnA-, or RCAN1/CnA mRNA-injected larvae at stage 47. The size of each panel was $0.25 \mathrm{~mm}^{2}$. Bars $=100 \mu \mathrm{m}$. b Number of branching points counted in a $0.25-\mathrm{mm}^{2}$ area. There was a significant difference between RCAN1 and RCAN1/CnA, as well as between RCAN1 and the other groups $\left({ }^{*} \mathrm{p}<0.05\right)$. c In vivo

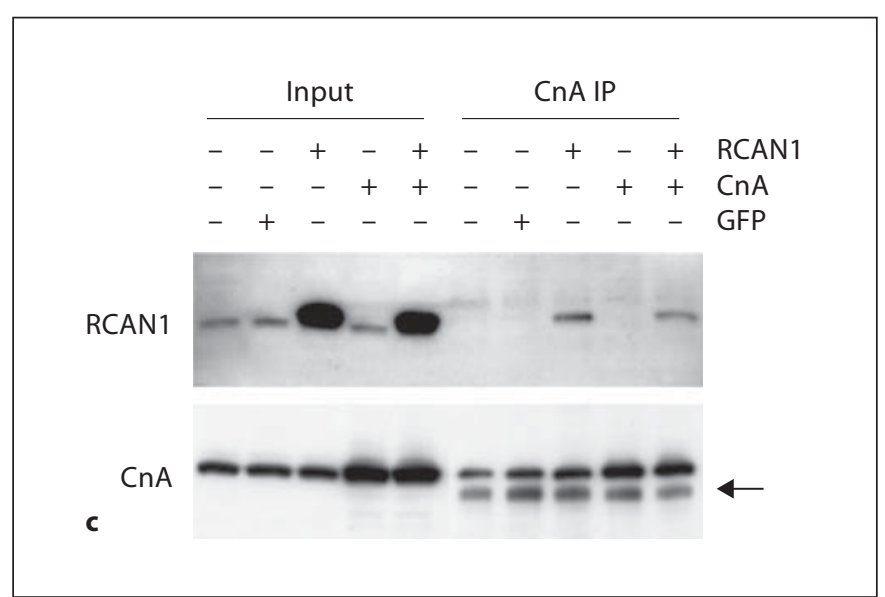

interaction of RCAN1 with CnA. Protein extracts were prepared from stage 10 embryos injected with the indicated mRNAs. CnA was pulled down with rabbit anti-pan-calcineurin A antibody. Input and IP samples were separated by SDS-PAGE, and both RCAN1 and CnA were detected by western blotting. The lower bands indicated by arrows in the CnA IP are the IgG heavy chain from the secondary antibody. function of RCAN1 in vivo. In the present study, we used $X$. laevis larvae for the in vivo functional analysis of RCAN1. This animal model is suitable for the study of complex blood vessel development because its vasculature can be visualized by a simple injection of dye, and genetic manipulation is easily available [29]. Using this in vivo model and red India ink as a powerful tool for imaging the complex vasculature by micorangiography, we found that overexpression of RCAN1 significantly reduced the number of branching points that subdivide a vascular zone into smaller regions. Considering the similarities in amino acid sequences of RCAN1 (fig. 5) and organogenesis of the vascular system between amphibi- ans and mammals [30], the negative effect of RCAN1 on amphibian vascular branching should also be conserved in mammals.

Baek et al. [31] recently showed the negative effect of RCAN1 on mammals by using Ts65Dn mice (segmental trisomy mouse model with a 1.7 -fold increase in RCAN1 protein expression) and transgenic mice with 3 copies of RCAN1 (a 2.4-fold increase in mRNA level). In these transgenic mice, the volume of the transplanted tumor as well as its microvessel density was significantly reduced. These inhibitory effects of RCAN1 on tumor angiogenesis support our results in X. laevis. Since endothelial cells isolated from the mice with 3 copies of RCAN1 demon- 
Fig. 7. RCAN1 suppresses vascular branching by interacting with calcineurin. $\mathbf{d ~ M i -}$ croangiography of the untreated, control, FK506-, and CsA- treated larvae at stage 47. Stage 46 larvae were exposed to $6 \mathrm{~h}$ of FK506 or CsA treatment (5, 50 and 100 nM) 3 times. Bars $=500 \mu \mathrm{m}$.
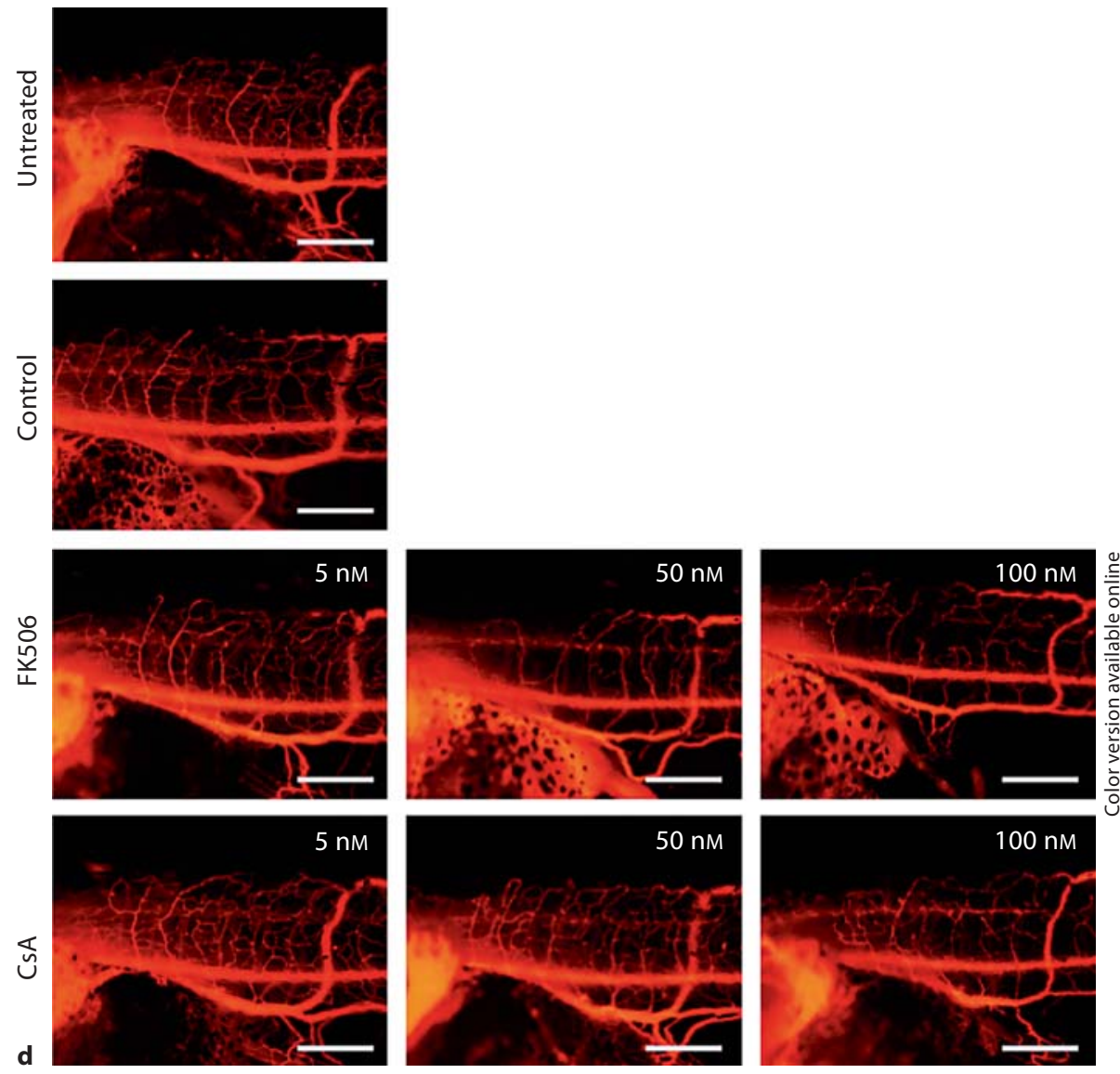

strated a decrease in VEGF sensitivity and had a suppressive effect on VEGF-induced proliferation, it is reasonable to hypothesize that the decrease in the number of vascular branching points may be caused by the loss of VEGF sensitivity in RCAN1.

As for the function of RCAN1 in mammalian angiogenesis, previous studies have reported conflicting results. In mice injected with a Matrigel plug with adenoviral vectors encoding RCAN1, a strong RCAN1 expression attenuated tube formation on the collagen gel [21]. Similarly, other functional analyses of RCAN1 overexpression also elucidated its inhibitory effect on cell migration, proliferation, and tube formation in cultured endothelial cells $[32,33]$. These results indicate the negative effect of RCAN1 on vascular formation. In contrast, loss-of-function studies of RCAN1 showed that tumor growth and microvessel density were significantly reduced in the RCAN1-null mouse [28] and that antisense oligonucleotides (ODNs) against RCAN1 suppressed the migration of endothelial cells as well as the development of angiogenesis [20]. These observations indicate the positive ef- fect of RCAN1 on vascular formation. Our finding that RCAN1 overexpression suppresses vascular branching supports the previously observed negative effect of RCAN1. Although loss-of-function studies could help to gain a more in-depth understanding of RCAN1 functions, in our study larvae injected with antisense MO against RCAN1 even at a low dose of $5 \mathrm{ng}$ /embryo developed gross malformations, prohibiting reliable analysis by microangiography. An explanation for the functional discrepancy of RCAN1 described above may be that the effect of RCAN1 differs with its expression level. Whether the effect of RCAN1 on angiogenesis is positive or negative is precisely predetermined. Specifically, a high level of RCAN1 expression has a negative effect, whereas a low level has a positive one. In the knockout or knockdown experiments, the complete depletion of RCAN1 by antisense ODNs probably did not evoke the weak signaling required for angiogenesis. During the normal development of X. laevis larvae in our study, the low level of RCAN1 expression became detectable at stage 41 and was followed by the start of the branching of microvessels in 
the trunk region at stage 44 . These observations agree with the notion that a low level of RCAN1 expression has a positive effect on vascular formation.

RCAN1 is an inhibitor of calcineurin-mediated signaling pathways and interacts directly and functionally with $\mathrm{CnA}$, the catalytic subunit of calcineurin [11]. In the present study, we showed that coexpressed $\mathrm{CnA}$, which binds to RCAN1 (fig. 7c), rescued the negative effects of RCAN1 on microvessel branching. Also, treatment with calcineurin inhibitors CsA and FK506 had negative effects on microvessel branching (fig. 7d). Previous studies reported that the effect of RCAN1 was also rescued by coinjection with adenovirus expressing constitutively active NFAT [21], which is a nuclear transcription factor activated by calcineurin [34-36]. Thus, it is highly possible that RCAN1 exerts its effect by inhibiting calcineurin-NFAT signaling.

In the present study, branching was normal in $\mathrm{CnA}$ mRNA-injected $X$. laevis larvae. This was expected because recombinant Xenopus CnA alone does not show any phosphatase activity [37]. Moreover, CnA has an autoinhibitory domain; by itself, the domain interacted with and inhibited elements of the catalytic sites of $\mathrm{CnA}$ during the quiescent state [38]. Binding of both the $\mathrm{CnB}$ subunit and $\mathrm{Ca}^{+} /$calmodulin to $\mathrm{CnA}$ is required to activate the sequestered catalytic $\mathrm{CnA}$ subunit [17]. Thus, overexpressed $\mathrm{CnA}$ alone did not alter the vascular morphology in our experiment. Therefore, overexpressed CnA may have ameliorated the negative effect of RCAN1 by binding to RCAN1, sequestering RCAN1 from endogenous calcineurin, and protecting calcineurin signaling from exogenous RCAN1 (fig. 7).

In conclusion, we focused on the effect of RCAN1 on the morphological variation among small and large vessels, not just the phenotypic variations we have previously reported [27, 39], and found that the overexpression of RCAN1 suppressed vascular branching. These results reveal a new role of RCAN1 in vascular branching and suggest the importance of RCAN1-calcineurin signaling for regulating morphologically different vasculatures.

\section{Acknowledgements}

This study was supported by a research grant from the Nippon Medical School Alumni Association, Nippon Medical School, Tokyo, Japan, and in part by a Grant-in-Aid for Young Scientists (B) (No. 20790168) from the Ministry of Education, Culture, Sports, Science and Technology of Japan.

\section{References}

1 Risau W: Mechanisms of angiogenesis. Nature 1997;386:671-674.

$\checkmark 2$ Fruttiger M: Development of the retinal vasculature. Angiogenesis 2007;10:77-88.

-3 Gerhardt H, Golding M, Fruttiger M, Ruhrberg C, Lundkvist A, Abramsson A, Jeltsch M, Mitchell C, Alitalo K, Shima D, Betsholtz C: VEGF guides angiogenic sprouting utilizing endothelial tip cell filopodia. J Cell Biol 2003; 161:1163-1177.

-4 Lee S, Jilani SM, Nikolova GV, Carpizo D, Iruela-Arispe ML: Processing of VEGF-A by matrix metalloproteinases regulates bioavailability and vascular patterning in tumors. J Cell Biol 2005;169:681-691.

5 Nakatsu MN, Sainson RC, Aoto JN, Taylor KL, Aitkenhead M, Perez-del-Pulgar S, Carpenter PM, Hughes CC: Angiogenic sprouting and capillary lumen formation modeled by HUVECs in fibrin gels: the role of fibroblasts and Angiopoietin-1. Microvasc Res 2003;66:102-112.

-6 Sainson RC, Aoto J, Nakatsu MN, Holderfield M, Conn E, Koller E, Hughes CC: Cellautonomous notch signaling regulates endothelial cell branching and proliferation during vascular tubulogenesis. FASEB J 2005;19:1027-1029.
Fuentes JJ, Pritchard MA, Planas AM, Bosch A, Ferrer I, Estivill X: A new human gene from the Down syndrome critical region encodes a proline-rich protein highly expressed in fetal brain and heart. Human Mol Genet 1995;4:1935-1944.

8 Miyazaki T, Kanou Y, Murata Y, Ohmori S, Niwa T, Maeda K, Yamamura H, Seo H: Molecular cloning of a novel thyroid hormoneresponsive gene, ZAKI-4, in human skin fibroblasts. J Biol Chem 1996;271:14567-14571.

-9 Yao YG, Duh EJ: VEGF selectively induces Down syndrome critical region 1 gene expression in endothelial cells: a mechanism for feedback regulation of angiogenesis? Biochem Biophys Res Commun 2004;321:648656.

10 Hesser BA, Liang XH, Camenisch G, Yang S, Lewin DA, Scheller R, Ferrara N, Gerber HP: Down syndrome critical region protein 1 (DSCR1), a novel VEGF target gene that regulates expression of inflammatory markers on activated endothelial cells. Blood 2004; 104:149-158.

11 Fuentes JJ, Genesca L, Kingsbury TJ, Cunningham KW, Perez-Riba M, Estivill X, de la Luna S: DSCR1, overexpressed in Down syndrome, is an inhibitor of calcineurin-mediated signaling pathways. Hum Mol Genet 2000;9:1681-1690.
12 Fuentes JJ, Pritchard MA, Estivill X: Genomic organization, alternative splicing, and expression patterns of the DSCR1 (Down syndrome candidate region 1) gene. Genomics 1997;44:358-361.

13 Abe M, Sato Y: cDNA microarray analysis of the gene expression profile of VEGF-activated human umbilical vein endothelial cells. Angiogenesis 2001;4:289-298.

14 Kingsbury TJ, Cunningham KW: A conserved family of calcineurin regulators. Genes Dev 2000;14:1595-1604.

15 Flanagan WM, Corthesy B, Bram RJ, Crabtree GR: Nuclear association of a T-cell transcription factor blocked by FK-506 and cyclosporin A. Nature 1991;352:803-807.

16 Gorlach J, Fox DS, Cutler NS, Cox GM, Perfect JR, Heitman J: Identification and characterization of a highly conserved calcineurin binding protein, $\mathrm{CBP} 1 /$ calcipressin, in Cryptococcus neoformans. EMBO J 2000;19: 3618-3629.

17 Rothermel BA, Vega RB, Williams RS: The role of modulatory calcineurin-interacting proteins in calcineurin signaling. Trends Cardiovasc Med 2003;13:15-21 
18 Chang CP, Neilson JR, Bayle JH, Gestwicki JE, Kuo A, Stankunas K, Graef IA, Crabtree GR: A field of myocardial-endocardial NFAT signaling underlies heart valve morphogenesis. Cell 2004;118:649-663.

19 Graef IA, Chen F, Chen L, Kuo A, Crabtree GR: Signals transduced by $\mathrm{Ca}\left(2^{+}\right) /$calcineurin and NFATc3/c4 pattern the developing vasculature. Cell 2001;105:863-875.

20 Iizuka M, Abe M, Shiiba K, Sasaki I, Sato Y: Down syndrome candidate region 1 , a downstream target of VEGF, participates in endothelial cell migration and angiogenesis. J Vasc Res 2004;41:334-344.

-21 Minami T, Horiuchi K, Miura M, Abid MR, Takabe W, Noguchi N, Kohro T, Ge X, Aburatani $\mathrm{H}$, Hamakubo T, Kodama T, Aird WC: Vascular endothelial growth factorand thrombin-induced termination factor, Down syndrome critical region-1, attenuates endothelial cell proliferation and angiogenesis. J Bboil Chem 2004;279:50537-50554.

22 Kluetzman KS, Perez AV, Crawford DR: DSCR1 (ADAPT78) lethality: evidence for a protective effect of trisomy 21 genes? Biochem Biophys Res Commun 2005;337:595601.

-23 Nakatsu MN, Sainson RC, Perez-del-Pulgar S, Aoto JN, Aitkenhead M, Taylor KL, Carpenter PM, Hughes CC: $\operatorname{VEGF(121)~and~}$ vEGF(165) regulate blood vessel diameter through vascular endothelial growth factor receptor 2 in an in vitro angiogenesis model. Lab Invest 2003;83:1873-1885.

-24 Hasebe T, Hartman R, Fu L, Amano T, Shi YB: Evidence for a cooperative role of gelatinase A and membrane type-1 matrix metalloproteinase during Xenopus laevis development. Mech Dev 2007;124:11-22.
25 Stockmann C, Doedens A, Weidemann A, Zhang N, Takeda N, Greenberg JI, Cheresh DA, Johnson RS: Deletion of vascular endothelial growth factor in myeloid cells accelerates tumorigenesis. Nature 2008;456:814818.

26 Nieuwkoop DP, Faber J: Normal Table of Xenopus laevis. Amsterdam, North-Holland, 1994.

-27 Fujiwara M, Jin E, Ghazizadeh M, Kawanami O: Activation of PAR4 induces a distinct actin fiber formation via p38 MAPK in human lung endothelial cells. J Histochem $\mathrm{Cy}$ tochem 2005;53:1121-1129.

28 Ryeom S, Baek KH, Rioth MJ, Lynch RC, Zaslavsky A, Birsner A, Yoon SS, McKeon F: Targeted deletion of the calcineurin inhibitor DSCR1 suppresses tumor growth. Cancer Cell 2008;13:420-431.

29 Ny A, Koch M, Schneider M, Neven E, Tong RT, Maity S, Fischer C, Plaisance S, Lambrechts D, Heligon C, Terclavers S, Ciesiolka M, Kalin R, Man WY, Senn I, Wyns S, Lupu F, Brandli A, Vleminckx K, Collen D, Dewerchin M, Conway EM, Moons L, Jain RK, Carmeliet P: A genetic Xenopus laevis tadpole model to study lymphangiogenesis. Nat Med 2005;11:998-1004.

30 Ciau-Uitz A, Fernandez C, Patient R: Xenopus as a Model to Study Endothelial Development and Modulation. New York, Cambridge University Press, 2007.

-31 Baek KH, Zaslavsky A, Lynch RC, Britt C, Okada Y, Siarey RJ, Lensch MW, Park IH, Yoon SS, Minami T, Korenberg JR, Folkman J, Daley GQ, Aird WC, Galdzicki Z, Ryeom S: Down's syndrome suppression of tumour growth and the role of the calcineurin inhibitor DSCR1. Nature 2009;459:1126-1130.
32 Qin L, Zhao D, Liu X, Nagy JA, Hoang MV, Brown LF, Dvorak HF, Zeng H: Down syndrome candidate region 1 isoform 1 mediates angiogenesis through the calcineurinNFAT pathway. Mol Cancer Res 2006;4: 811-820.

33 Gollogly LK, Ryeom SW, Yoon SS: Down syndrome candidate region 1-like 1 (DSCR1L1) mimics the inhibitory effects of DSCR1 on calcineurin signaling in endothelial cells and inhibits angiogenesis. J Surg Res 2007; 142:129-136.

- 34 Yang TT, Chow CW: Transcription cooperation by NFAT.C/EBP composite enhancer complex. J Bboil Chem 2003;278:1587415885.

35 Macián F, López-Rodríguez C, Rao A: Partners in transcription: NFAT and AP-1. Oncogene 2001;20:2476-2489.

36 Molkentin JD, Lu JR, Antos CL, Markham B, Richardson J, Robbins J, Grant SR, Olson EN: A calcineurin-dependent transcriptional pathway for cardiac hypertrophy. Cell 1998;93:215-228.

37 Saneyoshi T, Kume S, Natsume T, Mikoshiba $\mathrm{K}$ : Molecular cloning and expression profile of Xenopus calcineurin A subunit(1). Biochim Biophys Acta 2000;1499:164-170.

38 Hashimoto Y, Perrino BA, Soderling TR: Identification of an autoinhibitory domain in calcineurin. J Biol Chem 1990;265:19241927.

39 Fujiwara M, Jin E, Ghazizadeh M, Kawanami O: Differential expression of protease-activated receptors 1,2 , and 4 on human endothelial cells from different vascular sites. Pathobiology 2004;71:52-58. 